\title{
Momentum dependence of the topological susceptibility and its derivative at zero momentum with overlap fermions
}

\author{
Yoshiaki Koma*t \\ Numazu College of Technology, Numazu 410-8501, Japan \\ E-mail: koma@numazu-ct.ac.jp
}

\begin{abstract}
The derivative of the topological susceptibility at zero momentum is responsible for the validity of the Witten-Veneziano formula for the $\eta^{\prime}$ mass, and also for the resolution of the EMC proton spin problem. We investigate the momentum dependence of the topological susceptibility and its derivative at zero momentum using lattice QCD simulations with overlap fermions within quenched approximation. We expose the role of the low-lying Dirac eigenmodes for the topological charge density, and find the negative value for the derivative. While the sign of the derivative is consistent with the QCD sum rule in pure Yang-Mills theory, the absolute value becomes larger if only the contribution from the zero modes and the low-lying eigenmodes is taken into account.
\end{abstract}

8th Conference Quark Confinement and the Hadron Spectrum

September 1-6, 2008

Mainz. Germany

\footnotetext{
*Speaker.

$\dagger$ The author is partially supported by the Ministry of Education, Science, Sports and Culture, Japan, Grant-inAid for Encouragement of Young Scientists (B), 20740149, 2008, and the JSPS/DFG bilateral collaboration program. The author is grateful to E.-M. Ilgenfritz, K. Koller, G. Schierholz, T. Streuer, and V. Weinberg (QCDSF) for fruitful collaboration.
} 


\section{Introduction / Summary}

The derivative of the topological susceptibility $\chi\left(k^{2}\right)$ at zero momentum $\chi^{\prime}(0)$ is responsible for the validity of the Witten-Veneziano formula for the $\eta^{\prime}$ mass [1, 2]; the derivation of the formula assumes that the momentum dependence of the topological susceptibility $\chi\left(k^{2}\right)$ is moderate from $k^{2}=0$ to $m_{\eta^{\prime}}^{2}$, i.e. $\left|\chi^{\prime} m_{\eta^{\prime}}^{2}\right| \ll \chi$, as well as taking the large $N_{c}$ limit. This quantity is also helpful to disentangle the EMC proton spin problem [3]; the apparent small contribution of quark spin to the proton spin can be due to the small value of $\chi^{\prime}(0)$, which causes large suppression of the zeroth component of the axial charge $a^{0}$ relative to the eighth component $a^{8}$ in the first moment of the polarised proton structure function $g_{1}^{p}$ (see [4] for a review), which explains the violation of the Ellis-Jaffe sum rule [5] (or the OZI prediction of quark models).

We investigate the momentum dependence of the topological susceptibility and its derivative at zero momentum $\chi^{\prime}(0)$ using lattice QCD simulations with overlap fermions within quenched approximation. We find that $\chi^{\prime}(0)$ depends on the number of eigenmodes used to define the topological charge density (see Fig. 1); as the cutoff of eigenvalue of the Dirac operator increases, $\left|\chi^{\prime}(0)\right|$ decreases. In other words, $\left|\chi^{\prime}(0)\right|$ with the low-lying eigenmodes can be too large to accomodate the assumption used in deriving the Witten-Veneziano formula. This means that ultra-scale fluctuation of the topological charge density due to higher eigenmodes should also be taken into account, although it is often argued that the zero modes and low-lying eigenmodes play a relevant role for the topological structure of the QCD vacuum.

As discussed in refs. [6, 7, the topological charge density possesses global sign coherent structures, which get increasingly tangled as more and more eigenmodes are included, and the full topological charge density has a lower-dimensional laminar structure, together with a lumpy structure inside the sign coherent regions. After all, the vacuum is found to possess a multifractal structure. Such a complicated structure of the QCD vacuum is responsible for the behavior of $\chi^{\prime}(0)$.

\section{References}

[1] E. Witten, Nucl. Phys. B156, 269 (1979).

[2] G. Veneziano, Nucl. Phys. B159, 213 (1979).

[3] European Muon Collaboration, J. Ashman et al., Phys. Lett. B206, 364 (1988).

[4] G.M. Shore, Lect. Notes Phys. 737, 235 (2008), hep-ph/0701171.

[5] J.R. Ellis and R.L. Jaffe, Phys. Rev. D9, 1444 (1974).

[6] Y. Koma et al., PoS LAT2005, 300 (2006), hep-lat/0509164.

[7] E.-M. Ilgenfritz et al., Phys. Rev. D76, 034506 (2007), 0705.0018. 